\title{
El derecho a la portabilidad eléctrica. Análisis crítico del proyecto de ley chileno que lo establece
}

\section{The right to electrical portability. Critical analysis of the Chilean draft bill}

Rafael M. Plaza Reveco*

El artículo analiza el proyecto de ley chileno que establece el derecho a la portabilidad o migración eléctrica: esto es, el derecho de los usuarios finales del sistema de distribución a elegir a su suministrador de energía, mediante la separación del negocio de las redes de distribución del de la comercialización de la energía eléctri$c a$, un proyecto con que el legislador busca, en último término, reducir el poder monopólico de las distribuidoras de electricidad.

Palabras clave: Portabilidad eléctrica, distribución eléctrica, licitaciones de suministro eléctrico, comercialización de energía, contratos take or pay.
The paper delves on the Chilean draft bill that establishes the right to electrical portability, that is, the right of end users of the distribution system to choose their power supplier. The draft bill targets to separate the distribution networks business from that of sale of electric power. The lawmaker looks for opening competition and, ultimately, reducing the monopolistic market power of electricity distribution corporations.

Keywords: Electrical portability, power distribution, power supply tenders, energy sales, take or pay contracts.

\section{Introducción}

Desde la promulgación de la Ley General de Servicios Eléctricos (en adelante, LGSE)' , en 1982, los segmentos de generación y transmisión

\footnotetext{
* LLB/Grad.Dip. Universidad de Chile; LLM (NYU), PhD/LLM (UNIMELB), Post PhD (CUG). Dirección postal: Pío Nono N 1, Providencia, Santiago de Chile. Correo electrónico: rplaza@derecho.uchile.cl

1 Decreto con Fuerza de Ley $N^{\circ} 4 / 20018$ que fija el texto refundido, coordinado y sistematizado del Decreto con Fuerza de Ley No 1, de Minería, de 13 de septiembre de 1982, Ley General de Servicios Eléctricos. Ministerio de Economía, Fomento y Reconstrucción. Diario Oficial 05 Febrero de 2007.
}

Artículo recibido el 26 de mayo de 2021 y aceptado el 8 de noviembre de 2021. 
de energía han experimentado sucesivas e importantes reformas que -en lo sustancial- han abierto el mercado generador a la competencia y le distinguieron estructural, jurídica y económicamente del mercado de distribución de energía, reconocidamente monopólico.

Por contraste y hasta hoy, Chile no había abordado una reforma estructural del segmento de distribución que², por una parte, reforzare el desarrollo de las líneas; y por otra, regulare las condiciones del servicio a los usuarios abriendo para estos la opción de elegir a su suministrador.

El objetivo de este trabajo es presentar el proyecto de ley chileno que establece el llamado derecho a la portabilidad eléctrica: esto es, el derecho de los usuarios finales del sistema de distribución a elegir a su suministrador de energía ${ }^{3}$. Empleando una metodología exegética con enfoque jurídico-económico, extraerá sus principios fundamentales, efectuará el análisis crítico de sus disposiciones más relevantes y realizará un pronóstico sobre su integración sistémica a la LGSE y la eficacia de su implementación para introducir competencia al segmento.

\section{Desarrollo. Análisis crítico}

\section{El proyecto de ley sobre portabilidad eléctrica}

Por Mensaje No 156-368, de 4 de septiembre de 2020, el Presidente de la República de Chile comunicó a la Cámara de Diputados del Congreso el inicio de un proyecto de ley que reforma la LGSE y establece el derecho a la portabilidad eléctrica.

El proyecto de ley se estructura en un artículo único y siete artículos transitorios. El artículo único introduce múltiples disposiciones nuevas y de carácter sustancial a la LGSE. Primeramente, incorpora a la LGSE un Título III Bis cuyas normas identifican y distinguen el área propiamente monopólica de las redes de distribución eléctrica, vinculada a la propiedad y operación de las líneas de distribución y demás infraestructura asociada; de otras áreas o actividades vinculadas a esas mismas redes pero susceptibles de ser competitivas, como es el caso de la comercialización de la energía distribuida. Así, el proyecto incorpora la figura del "comercializador" de energía eléctrica, que responde grosso modo al modelo de Consolidated Power Supplier esta-

\footnotetext{
2 Solo recientemente se efectuó la intervención puntual derivada de la Ley N N 21.194 (D.O. 21.12.2019) que rebaja la rentabilidad de las distribuidoras e hizo cambios al proceso tarifario.

${ }_{3}$ Mensaje Presidencial No 156-368, de 4 de septiembre de 2020, a la H. Cámara de Diputados del Congreso Nacional, con el que inicia un proyecto de ley que modifica la LGSE y establece el derecho a la portabilidad eléctrica. Boletín No 13.782-08 de la H. Cámara de Diputados. Fecha de ingreso: 9 de septiembre de 2020. Estado a la fecha (15 de junio de 2021): Primer trámite constitucional.
} 
dounidense; ${ }^{4}$ y el derecho complementario de los usuarios finales a elegir un suministrador licenciado de electricidad.

Enseguida, el proyecto de ley introduce a la LGSE un Título III Ter nuevo en que crea la figura del "gestor de información", como un organismo privado, licitado internacionalmente, e independiente que preste el servicio de gestión de la información garantizando el acceso controlado y simétrico a ella, proteja los datos de los clientes y facilite a los usuarios la elección del suministrador ${ }^{5}$.

En tercer término, el proyecto incorpora otros cambios al texto actual de la LGSE, los que analizaremos en profundidad en la sección que sigue ${ }^{6}$.

A la fecha el proyecto de ley sortea su primer trámite constitucional en la Cámara de Diputados del Congreso Nacional.

\section{Principios fundamentales}

El proyecto de ley que nos ocupa se estructura en torno a cuatro principios fundamentales que pasamos a explicar.

\section{a) Identificación de áreas competitivas vinculadas a redes de distribución}

Las reformas introducidas a la LGSE por la "Ley Corta I" y "Ley Corta II" abordaron el segmento de generación, al permitir el acceso a redes de distribución y al exigir a las comercializadoras de energía acreditar que un por-

\footnotetext{
${ }^{4}$ En Estados Unidos, la generación, transmisión, distribución y venta de electricidad están reguladas por entidades gubernamentales a nivel federal, estatal y local. La Federal Energy Regulatory Commission (FERC) regula generalmente la transmisión eléctrica entre Estados y las ventas de energía mayorista; mientras que los Estados regulan -con preferencia al nivel local- las redes de distribución y las ventas al por menor, las que pueden ser tradicionalmente reguladas o competitivas. Así, en un mercado minorista regulado los consumidores no pueden elegir quién genera su energía y deben comprarla a la empresa de servicios públicos del área. Los mercados minoristas competitivos, en cambio, permiten a los consumidores finales elegir entre proveedores competitivos. Estos mercados no solo han favorecido la generación independientes en 24 Estados de la Unión Americana (por ejemplo, Texas, califormia y gran parte de los estados del noreste de USA); sino que además, en 18 de ellos (como Arizona, California, Michigan, Montana y Oregón) y en Washington D.C., el contenido central del modelo Consolidated Power Supplier contempla opciones que permiten a usuarios residenciales y/o industriales elegir su proveedor minorista de electricidad y opciones de generación, por ejemplo, de energía renovable. Para más detalles, véase Lazar, J. (2016). Electricity Regulation in the US: A Guide. Second Edition. Montpelier, VT: The Regulatory Assistance Project. En http://www.raponline.org/knowledge-center/electricityregulation-in-the-us-a-guide-2. Para un breve recuento histórico, veáse National Renewable Energy Laboratory (NREL), Technical Report NREL/TP-6A20-67106 Competitive Electricity Market Regulation in the United States: A Primer (Diciembre 2016).

5 Ya sea una empresa distribuidora tradicional o un "comercializador", como veremos, en el caso de clientes "pequeños" y "medianos" según la nueva categorización de consumos que propone el proyecto de ley (en adelante, PDL).

6 Entre las materias más relevantes: la manera de integrar el derecho a la portabilidad eléctrica con la seguridad el suministro, la categorización de clientes (en tres tipos: pequeños, medianos y grandes) basada en umbrales de potencia contratada (menor a $20 \mathrm{~kW}$, entre 20 y $5.000 \mathrm{~kW}$ y más de $5.000 \mathrm{~kW}$, respectivamente), la garantía de suministro licitado a clientes (equivalentes a usuarios pequeños) con independencia de si hubieren optado o no por precios libres, y derogación del art. 120 LGSE sobre peajes de distribución.
} 
centaje de sus ventas proviene de generación renovable no convencional ${ }^{7}$. El segmento de transmisión no quedó a la zaga gracias a la Ley N²0.936.

El proyecto de ley se aboca, finalmente, a la esperada reforma estructural del segmento de distribución. Y uno de sus principios o ejes fundamentales es el de identificar -en el segmento- áreas o mercados asociados a redes de distribución susceptibles de operar bajo un régimen de competencia abierta lo que, se espera, redunde en beneficio directo de los usuarios finales ya sea a través de mejores precios o mejoras en la calidad y seguridad del servicio.

El legislador tiene claro que los distribuidores, además de su función natural: suministrar energía eléctrica, pueden emplear el acceso a sus instalaciones y/o desarrollar -vinculadas a ellas- una serie de mercados anexos (normalmente, servicios complementarios y/o de almacenamiento) que, económicamente, presentan una estructura de mercado y un funcionamiento diversos.

Tradicionalmente, el segmento de distribución ha englobado tanto la infraestructura necesaria (las instalaciones que constituyen la red de distribución) para llevar a cabo el suministro a clientes finales, como la comercialización de la energía a los mismos. Pues bien, el proyecto de ley termina con esta amalgama exclusivamente jurídica y opta por un criterio jurídico-económico que distingue la estructura monopólica per se del mercado de transporte de electricidad a nivel de distribución y que apunta al rol de desarrollo y gestión de infraestructura (categorizado por la LGSE, además, como servicio público de distribución) ${ }^{8}$ del mercado de venta de la electricidad (ya distribuida) cuya estructura no solo no es monopólica, sino que susceptible de funcionar -con los debidos resguardos regulatorios- de manera competitiva.

Como se podrá advertir, la finalidad de este eje (identificación de espacios competitivos) dentro de la concepción tradicional del segmento de distribución equivale a incorporar un grado mayor de flexibilidad a su marco regulatorio. Lo cual resulta no solo factible por los avances tecnológicos en el área, asociados ya no solo a las fuentes de generación eléctrica; sino que también justificable por la finalidad de promover la gestión de la demanda de energía por los usuarios.

La concreción práctica del principio en comento se manifiesta de dos formas: en primer lugar, flexibilizando el mecanismo de licitaciones de suministro, en el sentido de hacerlo compatible con la prerrogativa de elegir suministrador, asegurando al mismo tiempo la continuidad y seguridad del suministro. En segundo lugar, permitiendo la entrada de nuevos actores competidores al futuro liberalizado mercado de comercialización, lo que el

\footnotetext{
7 La Ley N 19.940, de 2004 ("Ley corta I"), reguló los sistemas de transporte de energía eléctrica, estableció un nuevo régimen de tarifas para sistemas eléctricos medianos e introdujo adecuaciones a la LGSE que permitieron el acceso a las redes de distribución para generadores inferiores a 9 MW. La Ley No 20.257, de 2008 ("Ley corta II"), introdujo modificaciones a la LGSE con respecto a la generación de energía eléctrica con fuentes de energía renovables no convencionales. 8 Artículo $7^{\circ}$ LGSE.
} 
proyecto logra creando la nueva figura del "comercializador" de energía. El proyecto establece entonces, por un lado, los requisitos de ejercicio de la actividad, entre los cuales destaca su licenciamiento; y, por otro lado, sus características esenciales como: ser la contraparte comercial única del usuario final para el suministro y hacerle responsable del aseguramiento de la cadena de pagos por el uso de los sistemas de distribución, transmisión, servicios complementarios $\mathrm{u}$ otros relativos a usuarios finales?.

En resumen, el principio en comento busca aislar el carácter monopólico inmanente del negocio de transporte ${ }^{10}$ y abrir la competencia en la mayor cantidad de mercados específicos tradicionalmente vinculados a las redes. El efecto no solo es reducir el poder del monopolista, sino también empoderar al usuario final regulado ampliando su posibilidad de elección, otorgando mayor protección a sus datos y una mejor gestión de su consumo ${ }^{11}$.

\section{b) Definición y distinción de usuarios de distribución eléctrica basada en potencia conectada; y derogación de los actuales peajes de distribución}

Tradicionalmente, las distribuidoras se ocupan de la planificación e inversión en sus instalaciones, y operan y mantienen sus redes; pero también son las responsables de proporcionar el suministro comercializando la energía eléctrica a clientes libre y regulados y prestando servicios complementarios. Para el proyecto, estas áreas deberían poder ser desarrolladas competitivamente por alguien distinto a la distribuidora, por lo que introduce así la figura del "comercializador" de energía.

El proyectado nuevo artículo 122-1 de la LGSE definirá a los usuarios del sistema de distribución como "aquellas personas naturales o jurídicas que retiren o inyecten energía eléctrica en las instalaciones de distribución, o hagan uso de estas, tales como usuarios finales, operadores o explotadores de medios energéticos distribuidos, gestores en servicios en distribución, agregadores de generación o demanda, comercializadores de energía, prestadores de servicios complementarios o empresas generadoras" ${ }^{12}$.

¿Quiénes podrán interactuar con el nuevo comercializador? La respuesta es sencilla: los nuevos usuarios del sistema de distribución. El proyecto de ley abandona la tipología actual de clientes bajo la LGSE y la reemplaza por una nueva y triple categorización de clientes atendido su "tamaño": a) los llamados "clientes pequeños" con potencia conectada inferior a $20 \mathrm{~kW}$, b) los "clientes medianos", con potencia instalada entre $20 \mathrm{~kW}$ e inferior a 5.000 kW, y c) los "clientes grandes", con potencia instalada de 5.000 kW hacia

\footnotetext{
9 Artículo único $N^{\circ} 3$ del PDL, que introduce a la LGSE el nuevo artículo 122-3 Nº 5.

10 El negocio de las redes: asociado, normalmente, a la planificación e inversión en infraestructura (instalaciones de distribución, en este caso) y a su operación y mantenimiento (igualmente, en este caso, de las redes de distribución).

11 Como se verá, no enteramente por sí mismo por la intermediación del "gestor de información".

12 Art. único No3 del PDL. Incorpora a la LGSE el Título III bis que incluye el nuevo art. 122-1 sobre "Usuarios de los sistemas de distribución".
} 
arriba $^{13}$. Esta nueva categorización de clientes se explica en relación con la estructura de los mercados en que interviene cada categoría; y tiene su correlato práctico en las características asociadas a las licitaciones de suministro adecuadas para cada uno ${ }^{14}$, y también son relevantes en relación con la homologación de la titularidad y el ejercicio del derecho de portabilidad, que explicaremos en la sección siguiente ${ }^{15}$.

c) Derecho a la portabilidad o migración eléctrica (derecho a elegir al suministrador). Derechos complementarios y servicio de gestión de información

El proyecto de ley plantea incluir un nuevo artículo 122-2 a la LGSE, que detalla cuatro derechos de los usuarios finales. El primer numeral de dicho artículo establece el derecho de todos los "usuarios finales del sistema de distribución" a elegir su suministrador de energía y a cambiarlo sin costo alguno, asegurando su debida protección.

Pero, no todas las nuevas categorías de clientes podrán mantener el suministro provisto por una empresa distribuidora con la tarifa regulada conforme al procedimiento estándar, sino solo los clientes "medianos" y "pequeños"; opción que sigue vedada a los clientes "grandes", aunque conserven estos su facultad para contratar su suministro directamente con las empresas generadores (como en la actualidad).

Veamos cada una de las interacciones de comercialización posible que plantea el proyecto.

En primer lugar, la portabilidad -entendida como el derecho de poder elegir al suministrador- será una facultad enteramente nueva para los "clientes pequeños" quienes, de acuerdo a sus preferencias, podrán optar por el suministro de las distribuidoras a tarifa regulada o el ofrecido por un "comercializador" habilitado, de su elección. Su inclusión en el derecho a optar por la tarifa regulada u otras condiciones que pudiere ofrecerles el futuro "comercializador" -alternativa inexistente en la actualidad-es, sin lugar a dudas, un mejoramiento de la condición jurídica del cliente pequeño (por ejemplo, el cliente residencial). Lo que está por ver es si ello se traduce en un mejoramiento económico real del usuario final, con precios más bajos; o, al menos, en un mejoramiento de su condición comercial general por otras vías.

En segundo término, reconociendo la endémica dificultad de los "clientes medianos" de hoy para acceder a información transparente, completa y oportuna que ilumine mejor su decisión de suministro, el proyecto innova en darles la posibilidad de gestionarlo a través del "comercializador" de su preferencia con más el servicio y resguardo que les proporcionaría la nueva

\footnotetext{
13 Artículo único No 14 letra a) del PDL. Incorpora a la LGSE un artículo 147 nuevo sobre clasificación de usuarios finales.

${ }^{14}$ Vid infra sección E.

15 Vid infra sección D.
} 
figura del "gestor de información". En este sentido, se busca empoderar a una gama variada de consumidores de mediano tamaño, con posibles preferencias diversas sobre la fuente generadora, con diferencias de consumo estacional o con sesgos horarios, etc. Con respecto a este segmento de usuarios no cabe duda, entonces, que el proyecto plantea un mejoramiento de su capacidad efectiva de elección (al añadir a los "comercializadores"), pero también de que esta resulte potencialmente más beneficiosa en lo comercial, en tanto realmente sea precedida de mayor y mejor información sobre cada una de las alternativas disponibles de suministro.

Por último, los "clientes grandes" -según les denomina el proyecto o "libres" según la normativa vigente- siguen habilitados para contratar por sí mismos su suministro en una negociación libre con las generadoras, y son equiparados a los demás en cuanto a que también podrán acceder a un "comercializador" si lo desean.

En definitiva, la única distinción subsistente entre estos tres tipos de nuevos clientes es que los de la tercera clase: los "grandes", carecen de la posibilidad de acceder a suministros con tarifas reguladas. Esto resulta entendible desde un punto de vista de política pública, porque el resguardo que provee la regulación se justifica para aquellos clientes de tamaño mediano y/o pequeño, quienes normalmente adolecen de asimetría en la información a que tienen acceso, lo que menoscaba o hace inexistente su capacidad de negociación; lo que, claramente, no es el caso de los segmentos donde se observan mejores condiciones de competencia ergo de libertad de precios, como en el segmento de clientes de mayor tamaño.

En resumen, el derecho a la portabilidad eléctrica alcanza a todos los usuarios pequeños y medianos y este derecho les habilita para optar entre las ofertas propuestas por los "comercializadores" de energía licitados; o contratar el suministro de una empresa distribuidora con tarifa regulada. La misma prerrogativa de emplear a un "comercializador" se reconoce a los clientes grandes, quienes conservan además su facultad de contratar su suministro directa y libremente con los generadores.

Como anunciamos, conjuntamente con el derecho a la portabilidad, los demás numerales del nuevo artículo 122-2 que plantea el proyecto otorgan a los usuarios finales del sistema tres derechos complementarios: 1. Recibir información oportuna, clara y transparente sobre precios y condiciones en que se efectúa el suministro, tener a disposición los datos de consumo, recibir atención comercial oportuna de parte del "comercializador" y contar con la seguridad y calidad del suministro por la distribuidora ${ }^{16} ; 2$. Poder reclamar

${ }_{16}$ El artículo 122-2 № 2 del PDL establece que, para hacer efectivo el derecho a "recibir información oportuna, clara y transparente" se deberá "disponer del equipamiento de medida apropiado de acuerdo a lo que determine la norma técnica respectiva". A su turno, el artículo 122-15 del PDL establece la figura del "gestor de información" como responsable de "almacenar y gestionar los datos que se obtengan de los procesos de medición de consumo y gestión de la red de distribución". 
ante la SEC la afectación de estos derechos; y 3. Los demás derechos que el ordenamiento les confiera ${ }^{17}$.

$\mathrm{Ni}$ el derecho a migrar ni los derechos complementarios referidos serían posibles sin la nueva figura del "gestor de información"18. Este tendrá la misión de almacenar y gestionar los datos de las mediciones de consumo y gestión de la red de distribución ${ }^{19}$, prestar el nuevo servicio público de gestión de información ${ }^{20}$, y elaborar los balances económicos que servirán de base al Coordinador Eléctrico Nacional para realizar las transferencias económicas respectivas entre los agentes del mercado ${ }^{21}$. Así, entre sus principales características destacará su independencia reforzada por su giro exclusivo y vinculado al tratamiento de información relevante del segmento. Los usuarios finales tendrán libre acceso a una plataforma donde podrán comparar -uniformemente, ha de entenderse- las variadas tarifas que ofrezcan los "comercializadores".

En relación con la función de realizar balances económicos, se espera del "gestor" que -atendida su vinculación permanente con los "comercializadores" y el flujo de información (datos físicos de los usuarios, volúmenes de energía y/o potencia y horas) que recolecte de ellos pueda, primero, fijar el cargo a pagar por los usuarios por el uso de redes; y luego, entregar información agregada al Coordinador Eléctrico, quien realizará las transferencias económicas que correspondan. En este asunto, la Asociación Gremial que aúna a las empresas eléctricas ha manifestado dudas acerca de la capacidad del nuevo "gestor" para ocuparse de cuestiones financieras sin un claro deslinde con las atribuciones del tipo ya asignadas al Coordinador Eléctrico ${ }^{22}$.

Con respecto a las mediciones de consumo es necesario referir, brevemente, el estado de recambio de los Sistemas de Medición, Monitoreo y Control -vulgarmente conocidos como "medidores inteligentes"- tras la entrada en vigor de la Ley $N^{\circ} 21.076$, por ser la base del servicio que prestará el "gestor de información". Ello resultaría un avance no solo en transparencia de la información comercial consolidada y la gestión de demanda, sino también en la protección de los datos personales y de consumo de los usuarios, en el mejor de los casos.

\footnotetext{
17 Artículo 122-2 Nos 2, 3 y 4 del PDL.

18 De acuerdo con el proyecto de ley, el "gestor de información" será nominado mediante una licitación pública internacional dirigida por la Comisión Nacional de Energía (CNE). Vid art. 12217 del PDL.

19 La norma técnica que fija las características de los Sistemas de Medición, Monitoreo y Control es la Resolución Exenta Nº 468, de 12 de agosto de 2019, de la Comisión Nacional de Energía.

20 Artículo 122-18 del PDL.

21 Artículo 122-14 inciso final, del PDL

22 Asociación Gremial de Empresas Eléctricas A.G. En https://www.electricas.cl/ Véase Seminario web sobre Portabilidad Eléctrica, evento organizado por el Ministerio de Energía, la Comisión Nacional de Energía y el Comité Chileno del CIGRE, junto al patrocinio de la Asociación de Empresas Eléctricas, ACEN y ACERA, realizado los días 24, 25 y 28 de septiembre de 2020. Véase también EBP Chile, Empresas Eléctricas A.G. e IESD, Estudio: El futuro de la distribución eléctrica en Chile. ¿Hacia dónde vamos? (Septiembre 2020), pp. 95.
} 
Sin embargo, para que todo esto se implemente apropiadamente aparece necesario un estudio de costos, liderado por la Comisión Nacional de Energía, que defina procesos de gestión, plazos, procedimientos de seguridad para el manejo de información; y, además, normas complementarias que deslinden sus funciones financieras y aborden los costos del servicio, incluida la remuneración del "gestor" y los obligados a su pago, más aún, por las características monopólicas que algunos reconocen en él ${ }^{23}$.

d) Provisión segura y permanente de suministro. Las mejoras al sistema de licitaciones de contratos de suministro

La experiencia con las licitaciones de suministro demuestra que estas han -efectivamente- alentado el ingreso de nuevos actores al mercado y el desarrollo de numerosos nuevos proyectos eléctricos, han fomentado una mayor competencia e inversión, propiciado ofertas con precios de suministro cada vez más bajos, y provocado también -al menos- cierta disminución indirecta de los precios regulados. Pero, la misma experiencia también ha puesto de relieve el efecto que tienen la duración y el volumen de energía y potencia involucrados en los contratos de suministro a licitar, así como el efecto de estipulaciones que obstaculizan la migración de clientes ante fluctuaciones del mercado, o la búsqueda de mejores precios y/o mayor calidad de servicio. Todos, factores que a fin de cuentas terminan por rigidizar y ralentizar el traspaso de los beneficios del sistema de licitaciones a las tarifas reguladas.

El proyecto de ley se hace cargo de las "rigideces" del marco actual de licitaciones y propone modificaciones alineadas con los objetivos de profundizar la búsqueda de precios competitivos y flexibilizar el sistema de licitaciones para favorecer el traspaso de sus beneficios teóricos (precios más bajos) al precio real de suministro (especialmente, el precio regulado) ${ }^{24}$.

Como se anunció, aquí toma importancia y se justifica mejor la nueva categorización de los usuarios del sistema de distribución, pues -precisamente para conjurar tales rigideces- el proyecto de ley contempla como nueva obligación de la Comisión Nacional de Energía (en adelante, CNE): conformar un portafolio óptimo de contratos con modalidades de corto, mediano y largo plazo.

En otras palabras, en su consideración de las condiciones de mercado necesarias para determinar las bases de las licitaciones de suministro la CNE deberá -en el futuro y de acuerdo al proyecto- atender a tres tipos de previsión de demanda de energía: 1. una energía base (segura o suficiente) dirigida a precaver los consumos de los clientes "pequeños", por lo mismo, sujeta a mayores condiciones de anticipación del proceso licitatorio, suficiencia de volumen, y duración de los contratos a licitar; 2. energía variable, enfocada

23 BuCHMANN, M. (2017). Governance of data and information management in smart distribution grids: Increase efficiency by balancing coordination and competition. Utilities Policy, 1-10.

${ }^{24}$ Adicionalmente, la reconfiguración de las licitaciones permite explicar también el fin de los "peajes de distribución" del artículo 120 de la LGSE actual. 
en los requerimientos de los clientes "medianos" en la medida en que hayan optado por el régimen de precios regulados; y, finalmente, 3. la energía de déficit, necesaria para hacer ajustes de corto plazo.

Al reformar de esta manera el sistema actual de licitaciones se producirá igualmente un correlato en los contratos originados en él. En este punto y en lo que se refiere a energía base y energía variable principalmente, el proyecto dará pie a que se incorpore como requisito de los contratos -en las bases de las licitaciones- cláusulas take or pay (en adelante, ToP), esto es, aquellas en virtud de las cuales el suministro de la energía contratada se ha de pagar a todo evento, con independencia de su consumo efectivo; y un orden de despacho de (la energía asociada a) los contratos con un criterio cuya determinación el proyecto entrega a un reglamento.

Como se puede colegir, esta propuesta de reconfiguración de las licitaciones tiene incidencia directa con la disponibilidad y seguridad del suministro de energía, según el tipo de cliente y el período relevante de análisis económico; así como, jurídicamente, en la duración de los contratos.

3. Análisis crítico de aspectos clave del proyecto de portabilidad eléctrica: barreras de entrada, justificación, transición y equilibrio económico de los contratos y contenido real de la homologación de usuarios

Tratándose de un proyecto de ley complejo y reciente las interrogantes que surgen son numerosas; pero, dedicaremos esta sección solo a cinco especialmente significativas. La primera se relaciona con la justificación de un proyecto de esta envergadura. Más allá de la intervención puntual de la Ley $N^{\circ} 21.194^{25}$, el legislador no había acometido una reforma estructural del segmento de distribución. El objetivo manifiesto del proyecto es que los usuarios finales del sistema puedan obtener precios de energía más bajos, ofertas diferenciadas y personalizadas, y mejor calidad en la atención comercial.

La crítica a la necesidad de acometer esta reforma para obtener aquellos fines es válida, si se atiende al hecho que poner en marcha el nuevo esquema de comercialización de energía supone un cambio de peso, más aún si se hubiera podido plantear una alternativa legislativa menos drástica, como podría ser -simplemente- liberalizar a un sector más amplio de usuarios finales, con una reducción del límite actual de 500 kW, por ejemplo. De esta forma también se habría permitido a nuevos actores acceder a suministros libres con la opción de retornar al suministro regulado.

Es posible que, bajo el nuevo esquema de comercialización que plantea el proyecto, la migración de clientes que están en el rango intermedio -quienes tendrán la opción de seguir siendo abastecidos por las empresas distribuidoras (a precios de regulación) o de emplear un comercializador (quien podría asegurarles el suministro en mejores condiciones de precio y/o de servicio)- vaya a provocar dicho efecto en los precios regulados. Con el sistema

${ }^{25}$ Ley Nº 21.194 (D.O. 19.12.2019), que rebajó la rentabilidad de las distribuidoras. 
actual de comercialización, esta afectación del precio regulado no se da en la práctica, porque el efecto de la migración del segmento de consumidores medianos (entre $500 \mathrm{~kW}$ y menos de $5 \mathrm{MW}$ ) se expresa, más bien, a través de sobrecontratación y/o reducción de los volúmenes de energía expresados en los contratos licitados, pero no por vía de cambios en los precios.

Es factible, además, que la reforma permita a usuarios finales pasar de un proveedor de suministro a otro con estructuras de precio distintas ("comercialización regulada" 26 o "comercialización libre"27); lo que implica previsiones de suministro distintas (energía segura, variable o deficitaria), que terminen impactando favorablemente el nivel de los precios regulados. Esto, precisamente, porque en el esquema actual la transparencia, compleción y oportunidad de la información, así como el poder de negociación de un cliente solo, que migra de regulado a libre, no están en absoluto asegurados. El proyecto, en cambio, deposita su fe en que tales asimetrías de información, diferencias de poder y demás fallas de mercado sean conjuradas por la labor del "gestor de información" en beneficio de los usuarios, por vía del comercializador.

La segunda gran interrogante que plantea el proyecto apunta al objetivo de atraer competencia a un nuevo mercado relevante detectado y separado, a través de la figura del comercializador y la necesidad de imponer requisitos de ejercicio a la actividad de comercializar energía eléctrica, cuya definición contempla el nuevo artículo $122-3$ inc. $1^{\circ}$ del proyecto ${ }^{28}$. Lo que resulta criticable desde el punto de vista jurídico-económico, pues imponer requisitos de ejercicio a una actividad equivale a levantar "barreras de entrada" a la misma, lo que resulta no solo contra intuitivo sino potencialmente contraproducente. No obstante, en el caso de industrias reguladas como la eléctrica aparece justificable por la necesidad imperiosa de asegurar la cadena de pagos, sobre todo si se acuerda al "comercializador" el rol de contraparte única. El proyecto de ley contempla una serie de requisitos habilitantes de distinto tipo para obtener una licencia de comercialización de energía eléctrica: administrativos (cierta organización societaria y garantías de cumplimiento normativo), de solvencia financiera (como garantía de participación estable en el mercado), relativos a la protección del usuario (vinculados a condiciones de marketing e información completa y oportuna del consumidor) y relacionados con el aseguramiento de la cadena de pagos (especialmente, en relación con las disposiciones del Reglamento de Coordinación y Operación). A pesar de estos resguardos -que algunos podrán juzgar razonables y objetivos- el riesgo de discriminación de entrada no es conjurado del todo.

\footnotetext{
26 Artículo 122-4 del PDL. La comercialización regulada es caracterizada como servicio público.

27 Art. 122-5 del PDL. La comercialización libre es la que efectúa el comercializador habilitado con licencia respectiva a los usuarios finales que lo soliciten y dentro de su área de operación.

28 Dicho artículo indica: "La comercialización de energía eléctrica consiste en la compra y venta de energía y potencia en un sistema eléctrico. Los comercializadores habilitados podrán suministrar a usuarios finales solo a través del establecimiento de contratos con estos últimos".
} 
La tercera interrogante es cómo, finalmente, se compatibilizarán los contratos licitados actuales de suministro con la labor del nuevo comercializador por una parte; y con los cambios propuestos al régimen de licitaciones, por otro. En este sentido, la crítica radica en que las disposiciones transitorias del proyecto no son del todo explícitas en lo que a la situación de los contratos actuales se refiere dejando demasiados asuntos de importancia a la potestad reglamentaria 29 , lo que favorece la incertidumbre y la desconfianza de los actores del mercado. Así, por ejemplo, la Asociación Chilena de Energías Renovables y Almacenamiento, A.G. ha expresado su preocupación sobre el debido equilibrio que debería existir entre los contratos de suministro licitados antes y después de la portabilidad dejando ver -con ello- que las normas de transición del proyecto de ley deberían haber sido explícitas en cuanto a asegurar que el balance económico de los contratos no se vería afectado. La aprensión parece verse sustentada en la norma por la cual la CNE "podrá diseñar los procesos de licitación de manera tal que el suministro requerido pueda ser cubierto mediante diferentes tipos de contrato de largo y mediano plazo conjuntamente, los cuales podrán incluso competir por el mismo suministro licitado"; 30 y, además, por la norma en virtud de la cual la metodología que establezca el orden de mérito para el despacho de la energía asociada a los distintos tipos de contratos licitados para el suministro de energía base, energía variable y energía deficitaria queda, igualmente, a merced de la discrecionalidad de la autoridad reglamentaria ${ }^{31}$.

Pues bien, si se tiene en cuenta que una virtud que se espera de las regulaciones eléctricas es que -dentro de lo posible- sean flexibles, esto es, que permitan el espacio adecuado a los vertiginosos cambios de la industria y de los mercados contemporáneos, la crítica puede retrucarse. En efecto, si bien es cierto que la técnica legislativa del proyecto parece apoyarse en demasía en la capacidad técnica y prescindencia de la autoridad regulatoria; no lo es menos que el proyecto mismo sienta los lineamientos principales a los que deberá someterse la reglamentación de ejecución. Un ejemplo de esto se aprecia al disponer el proyecto requisitos mínimos para las interacciones entre los usuarios pequeños y medianos y los comercializadores. El proyecto de ley entrega su desarrollo a una norma reglamentaria, pero -a la vez- establece parámetros adecuados, mencionando entre ellos las cláusulas admisibles de los contratos, la duración máxima de los mismos, la actualización de los precios, alternativas de pago o prepago, estructura tarifaria y derechos y deberes sobre manejo y protección de información sobre los usuarios finales ${ }^{32}$.

La cuarta crítica o interrogante que puede formularse al proyecto reside en que la nueva estructura de comercialización descansa, por una parte, en la

\footnotetext{
29 De acuerdo con el art. $1^{\circ}$ transitorio del PDL, los reglamentos de ejecución necesarios deberán dictarse dentro del plazo de dieciocho meses contados desde la publicación de la ley en el Diario Oficial.

30 Art. 122, nuevo art. 135 bis inciso $6^{\circ}$.

31 Art. único $N^{\circ} 11$ del PDL, que introduce a la LGSE un nuevo art. 135 bis.

32 Art. único $\mathrm{N}^{\circ} 3$ del PDL, que incorpora a la LGSE un nuevo art. 122-3 inciso $2^{\circ} \mathrm{N}^{\circ} 1$.
} 
introducción de la figura del "comercializador"; y, por otra, en la homologación de los usuarios del sistema de distribución para acceder a los servicios de aquel, con la posibilidad -para aquellos "medianos" y "pequeños"- de optar por seguir siendo suministrados bajos precios regulados a nivel de distribución. Así las cosas, sin una estructura aglutinante de esos clientes "medianos" y "pequeños" que garantice una gestión común de su demanda frente a un comercializador escogido, el acceso homologado a sus servicios bien podría devenir ineficaz (en términos de asegurarles precios de suministro más bajos) por una parte; y, por otra, reducir su nuevo estatus homologado a la retórica libertad de poder seguir simplemente como están: vinculados a un precio regulado.

Por último, y especialmente desde la óptica de los participantes en el mercado actual con costos más bajos, se plantea la interrogante si los cambios a la comercialización y al sistema de licitaciones no encubren, en realidad, el lobby del sector de la industria que intentaría proteger el despacho de contratos de suministro de larga data a los que se asocia, además, energía cara.

El origen de la idea se remonta a las mismas falencias o rigideces del sistema de licitación de suministro que ha sido incapaz, con la excepción de ciertos componentes regulados, de obtener una rebaja generalizada de los precios. Si se recuerda, esta rigidez del proceso se atribuye tanto al tamaño y duración de los contratos como al efecto de sobrecontratar volúmenes de energía. Bajo una combinación de estas circunstancias, por ejemplo: altos volúmenes de energía comprometidos, largos plazos de duración de los contratos y la ausencia de un desincentivo real y efectivo al acaparamiento: como es incorporar la cláusula ToP en los contratos licitados, no resulta descabellado el que -salvo excepciones- los precios de suministro adolezcan de un inmovilismo excesivo; o que, en el peor escenario, los precios suban, con todo el efecto dominó sobre los componentes regulados de la tarifa a que está obligado el usuario final.

Según el régimen de transición en las disposiciones transitorias del proyecto, tanto la gradualidad como la implementación del procedimiento técnico y objetivo que determine el cronograma de entrada en vigencia de la ley, no solo requerirán un Decreto con Fuerza de ley como requisito de forma; sino que, además, este deberá considerar el informe de licitaciones del artículo 131 ter de la LGSE ${ }^{33}$.

Más aún, el proyectado nuevo artículo 135 bis de la LGSE dispone que, en relación con los contratos de largo plazo, las bases de licitación podrán contemplar una facturación mínima del suministro contratado, con indepen-

\footnotetext{
33 Informe que deberá disponer, entre otros: 1. Parámetros de ingreso a zonas de comercialización como sería, por ejemplo, establecer un límite a la demanda total de energía disponible para libre elección de los usuarios; y que expresamente contempla que deberá "resguardar el equilibrio económico de los contratos de suministro vigentes"; y 2. Una instancia de participación y presentación de observaciones tanto de terceros como de interesados en el proceso.
} 
dencia de la energía efectivamente consumida. En otros términos, el proyecto permite -a nivel de bases- modificar la estipulación ToP para habilitar un cobro mínimo, lo que se justifica, pues normalmente los contratos largos se asocian con inversión en obras de infraestructura.

Así el legislador ha tenido en cuenta y considerado, con gravedad y detalle suficientes, el problema de la convivencia entre los antiguos y los nuevos contratos de suministro, la incidencia del factor ToP y el equilibrio económico entre los suministros asociados a aquellos.

\section{Pronóstico de integración al sistema de la LGSE e implementación eficaz}

La tarea de intentar un pronóstico acerca de la integración e implementación de un marco normativo aún en proyecto podría parecer un ejercicio especulativo. No obstante, el intento se justifica y tiene utilidad si se lo plantea de una manera abstracta y enfocada en los que -con justicia- se podrían llamar los dos contenidos fundamentales del proyecto de portabilidad: el régimen nuevo de comercialización de energía y los cambios al régimen de licitaciones de contratos de suministro.

En cuanto a la integración al sistema de la LGSE, la tríada usuario final -"gestor de información"- "comercializador" no resulta disruptiva con la orgánica existente; antes bien, se espera que las dos nuevas figuras institucionales ("gestor de información" y "comercializador") llenen vacíos funcionales al mejoramiento de la competencia en el mercado relevante contrapesando el poder monopólico actual de las distribuidoras y fortaleciendo la desmedrada posición comercial de los consumidores finales.

En cuanto al pronóstico de su implementación, solo cabe observar que si ella no es exitosa -definiendo el fracaso de la misma como la ausencia de ofertas de suministro más baratas que las de las actuales empresas distribuidoras- simplemente los clientes no migrarán de régimen. Si los "comercializadores" no se posicionan como competidores reales de las distribuidoras -ya sea a través de precios más atractivos, mejor atención comercial y calidad de su servicio, más diversificación y personalización de productos- la portabilidad no habrá tenido sentido. Por el bien de los consumidores finales, se espera lo contrario.

Licitar contratos para suministro es un rubro en que tanto la autoridad como la industria chilena poseen dilatada experiencia, por lo que la integración normativa a la LGSE no debería presentar problemas de importancia. Muy distinto es el caso, de los problemas que es posible prever con la complementación reglamentaria de algunas de las normas que contempla el proyecto, como por ejemplo la que dice relación con el orden de despacho de los contratos; u otra no menos controversial y relevante como la de la licitación internacional del proyectado artículo 122-1734.

${ }^{34}$ Incorporado por el artículo único No3 del PDL y relativo a la licitación internacional del "gestor de información". 
Aunque los mejoramientos introducidos al régimen de licitaciones resultan bien encaminados en términos generales y sea plausible pronosticar que esta apertura generará más competencia en el mercado; no es igual de sencillo especular que ello tendrá -necesaria y automáticamente- el tan anhelado efecto de rebaja en los precios ${ }^{35}$, y la razón de la cautela radica en que nada se ha investigado del no menos plausible aumento de los costos de transacción al introducir figuras institucionales nuevas. Así, resulta demasiado pronto para prever el efecto neto entre una mayor flexibilidad y apertura a la competencia en las licitaciones bis-a-bis un eventual aumento de los costos de transacción en la comercialización.

\section{Conclusiones}

El proyecto de ley identifica el núcleo de actividad propiamente monopólica de la distribución: el vinculado a la propiedad, operación y mantenimiento de las instalaciones; y lo separa de la comercialización de la energía, actividad que el legislador estima susceptible de funcionamiento competitivo, bajo los principios y reglas analizadas.

Con dicha separación, el legislador espera abrir la competencia en áreas susceptibles del segmento, atraer nuevos actores e inversiones en infraestructura, homologar el derecho del usuario final a elegir a su proveedor y conseguir una rebaja general en los precios a nivel de distribución.

Para lograrlo, el proyecto reclasifica a los usuarios finales del sistema de distribución, incorpora las figuras del "comercializador" y del "gestor de información" y se embarca en modificaciones a las licitaciones de contratos de suministro en atención a una nueva y triple categorización basada en la previsión de la demanda de energía (energía base, variable y deficitaria) para los usuarios definidos.

El telón de fondo en que se tramitará el proyecto está determinado no solo por el estado actual de la LGSE, sino particularmente por dos circunstancias fácticas de importancia: los contratos de suministro vigentes, por una parte; y el estado de la tecnología en el segmento de distribución, por la otra. En efecto, la transición y el trato a los contratos vigentes configuran un problema importante para la viabilidad del proyecto, pues este deberá resolver sobre el equilibrio económico entre aquellos y los contratos a que den origen las nuevas normas sobre licitaciones. A su turno, la controversia sobre el recambio de medidores digitales regulado por la Ley $N^{\circ} 21.076$ incide sobre la utilidad y efectividad de la función del "gestor de información".

Así las cosas, la reforma estructural del segmento de distribución aparece justificada más por el avance tecnológico de la industria y sus potencialidades, que por la certeza de alcanzar su fin manifiesto de obtener precios de

35 Aunque ello resulta teóricamente plausible si se tratase de un mercado perfectamente competitivo. 
energía eléctrica más bajos a través de mayor apertura de competencia. Está por verse cuál será el efecto neto de otorgar mayor flexibilidad a las licitaciones y añadir costos de transacción a la comercialización.

Sin perjuicio de lo dicho, es plausible que la esperada reducción de precios pueda ocurrir -al menos en lo que a precios regulados se refiere- si el efecto de la migración o portabilidad de un conglomerado mayor de usuarios se combina con el de los cambios a las licitaciones de suministro, particularmente, en lo que atañe a las de energía base y variable, por estar estas más vinculadas a proyectos de infraestructura y contratos de suministro de largo y mediano plazo que los sustentan, en la medida en que el reglamento respectivo resuelva -prontamente y de buena manera- la transición y la coexistencia (orden de despacho) de los contratos licitados antiguos y nuevos.

El nuevo mecanismo de comercialización, sin duda, tiene la ventaja de homologar a los clientes en su derecho a elegir su suministrador de energía y la de erigirse como contraparte única frente al usuario -a la vez- inserto en y responsable de la cadena de pagos del sistema. Sin embargo, aún no resulta del todo claro cómo esta nueva figura podrá conjurar los casi inevitables mayores costos de transacción que supone un intermediario económico.

Finalmente, el proyecto tiene un efecto potencial poderoso: reducir el poder de mercado de las empresas distribuidoras -hasta hoy- monopolistas. Consecuencialmente, el efecto de empoderar al usuario final regulado no solo otorgándole la posibilidad de elegir al suministrador con pleno resguardo de su opción de retornar al universo de precios regulados; sino que, además, ampliando la posibilidad de gestionar de manera más eficiente su propia demanda.

\section{Bibliografía citada}

BuchmanN, M. (2017). Governance of data and information management in smart distribution grids: Increase efficiency by balancing coordination and competition. Utilities Policy, 1-10.

eBP Chile, Empresas Eléctricas A.G. e IESD, Estudio: El futuro de la distribución eléctrica en Chile. ¿Hacia dónde vamos? (Septiembre 2020), pp. 95.

LAZAR, J. (2016). Electricity Regulation in the US: A Guide. Second Edition. Montpelier, VT: The Regulatory Assistance Project. En http://www.raponline.org/knowledge-center/ electricityregulation-in-the-us-a-guide- 2 .

National Renewable Energy Laboratory (2016), Technical Report NREL/TP-6A20-67106 Competitive Electricity Market Regulation in the United States: A Primer (Diciembre 2016).

\section{Normativa citada}

Constitución Política de la República de Chile [Const.]. 17 de septiembre de 2005 (Chile).

Ley N ${ }^{\circ} 19.940$ de 2004. Regula Sistemas de Transporte de energía eléctrica, establece un nuevo régimen de tarifas para sistemas eléctricos medianos e introduce las adecuaciones que indica a la Ley General de Servicios Eléctricos.

Ley $N^{\circ} 20.018$ de 2005. Modifica el marco normativo del sector eléctrico. 9 de mayo de 2005. D.O. N 38.165 . 
La Ley N²0.257, de 2008 ("Ley corta II"), introduce modificaciones a la Ley General de Servicios Eléctricos con respecto a la generación de energía eléctrica con fuentes de energía renovables no convencionales.

Ley $\mathrm{N}^{\circ} 20.936$ de 2016. Establece un nuevo sistema de transmisión eléctrica y crea un organismo coordinador independiente del sistema eléctrico nacional. 11 de julio de 2016. D.O. No 41.517.

Ley $N^{\circ} 21.076$ de 2018. Modifica la ley general de servicios eléctricos para imponer a la empresa distribuidora de energía la obligación de solventar el retiro y reposición del empalme y medidor en caso de inutilización de las instalaciones por fuerza mayor. 19 de febrero de 2018. D.O. N 41.994.

Ley $N^{\circ} 21.194$ de 2019. Rebaja la rentabilidad de las empresas de distribución y perfecciona el proceso tarifario de distribución eléctrica. 17 de diciembre de 2019. D.O. $\mathrm{N}^{\circ} 42.534$.

Decreto $N^{\circ} 4 / 20018$ [con fuerza de ley]. Fija texto refundido, coordinado y sistematizado del decreto con fuerza de ley $N^{\circ} 1$, de minería, de 1982, ley general de servicios eléctricos, en materia de energía eléctrica. 12 de mayo de 2006. D.O. № 38.079.

DS N ${ }^{\circ} 4 T$ [Ministerio de Energía]. Fija peajes de distribución aplicables al servicio de transporte que presenten las empresas concesionarias de servicio público de distribución de electricidad que se indican]. 1 de marzo de 2018.

Mensaje N ${ }^{\circ}$ 156-368 [Ministerio de Hacienda]. Informe financiero. Proyecto de Ley que establece el derecho a la portabilidad eléctrica. 7 de septiembre de 2020.

Mensaje N ${ }^{\circ}$ 283-368 [Secretaría General de la Presidencia]. Hace presente urgencia en el despacho del proyecto de ley que indica. 9 de septiembre de 2020.

Informe Final [Ministerio de Energía]. Proceso de Planificación Energética de Largo Plazo. 19 de febrero de 2018.

Resolución Exenta Nº 468 [Comisión Nacional de Energía]. norma técnica que fija las características de los Sistemas de Medición, Monitoreo y Control (SMMC). 12 de agosto de 2019.

Resolución exenta № 706 [Comisión Nacional de Energía]. Norma técnica de Calidad de Servicio para Sistemas de Distribución. 7 de Diciembre de 2017. 
